\title{
Mechanical Circulatory Support in Place at Start of Procedure
}

National Cancer Institute

\section{Source}

National Cancer Institute. Mechanical Circulatory Support in Place at Start of Procedure. NCl Thesaurus. Code C99981.

Mechanical Circulatory Support was in place at the start of the procedure. (ACC) 